\title{
Primary somatosensory cortex in chronic low back pain - a 'H-MRS study
}

This article was published in the following Dove Press journal:

Journal of Pain Research

17 May 20II

Number of times this article has been viewed

\author{
Neena K Sharma' \\ Kenneth McCarson ${ }^{2}$ \\ Linda Van Dillen ${ }^{5}$ \\ Angela Lentz' \\ Talal Khan ${ }^{3}$ \\ Carmen M Cirstea ${ }^{1,4}$ \\ 'Department of Physical Therapy and \\ Rehabilitation Science, ${ }^{2}$ Department \\ of Pharmacology, Toxicology and \\ Therapeutics, ${ }^{3}$ Department of \\ Anesthesiology, ${ }^{4} \mathrm{Hog}$ lund Brain \\ Imaging Center, University of Kansas \\ Medical Center, Kansas City, KS, USA; \\ ${ }^{5}$ Program in Physical Therapy and \\ Department of Orthopedic Surgery, \\ Washington University School of \\ Medicine, St Louis, MO, USA
}

\begin{abstract}
The goal of this study was to investigate whether certain metabolites, specific to neurons, glial cells, and the neuronal-glial neurotransmission system, in the primary somatosensory cortex (SSC), are altered and correlated with clinical characteristics of pain in patients with chronic low back pain (LBP). Eleven LBP patients and eleven age-matched healthy controls were included. N-acetylaspartate (NAA), choline (Cho), myo-inositol (mI), and glutamine/glutamate (Glx) were measured with proton magnetic resonance spectroscopy $\left({ }^{1} \mathrm{H}-\mathrm{MRS}\right)$ in left and right SSC. Differences in metabolite concentrations relative to those of controls were evaluated as well as analyses of metabolite correlations within and between SSCs. Relationships between metabolite concentrations and pain characteristics were also evaluated. We found decreased NAA in the left SSC $(P=0.001)$ and decreased Cho $(P=0.04)$ along with lower correlations between all metabolites in right SSC $(P=0.007)$ in LBP compared to controls. In addition, we found higher and significant correlations between left and right $\mathrm{mI}$ $(P<0.001$ in LBP vs $P=0.1$ in controls $)$ and between left $\mathrm{mI}$ and right $\mathrm{Cho}(P=0.048$ vs $P=$ $0.6)$. Left and right NAA levels were negatively correlated with pain duration $(P=0.04$ and $P=0.02$ respectively) while right Glx was positively correlated with pain severity $(P=0.04)$. Our preliminary results demonstrated significant altered neuronal-glial interactions in SSC, with left neural alterations related to pain duration and right neuronal-glial alterations to pain severity. Thus, the ${ }^{1} \mathrm{H}-\mathrm{MRS}$ approach proposed here can be used to quantify relevant cerebral metabolite changes in chronic pain, and consequently increase our knowledge of the factors leading from these changes to clinical outcomes.
\end{abstract}

Keywords: chronic low back pain, primary somatosensory cortex, magnetic resonance spectroscopy, neuronal-glial interactions

\section{Introduction}

The most prevalent and debilitating chronic pain condition is low back pain (LBP), affecting $85 \%$ of adults in US. ${ }^{1}$ Chronic LBP, defined by the presence of pain for longer than 3 months, is associated with significant physical and psychological disability that results in work absenteeism and economic burden, costing $\$ 86$ billion annually. ${ }^{2-4}$ Since the spinal imaging findings are poorly correlated with clinical characteristics of pain, ${ }^{5}$ brain imaging studies ${ }^{6-9}$ have attempted to gain a better understanding of pain neurobiology. Recently, the spectacular development of imaging techniques ${ }^{10-12}$ has improved this understanding even more.

Accumulating evidence suggests that chronic pain is due to central nervous system changes. ${ }^{6}$ Specifically, animal studies have shown spinal ${ }^{13,14}$ and cortical ${ }^{15,16}$ sensitization in various chronic pain conditions. Human imaging studies have also
Correspondence: Carmen M Cirstea Hoglund Brain Imaging Center, University of Kansas Medical Center, 390I Rainbow Blvd, Mail Stop I052, Kansas City, KS, USA, 66160

Tel + I 9135884373

Fax $+|913588907|$

Email ccirstea@kumc.edu 
revealed pathophysiological changes in brain regions involved in both affective-motivational and sensorial aspects of pain processing in chronic LBP. For instance, proton magnetic resonance spectroscopy $\left({ }^{1} \mathrm{H}-\mathrm{MRS}\right)$ studies have shown low N-acetylaspartate (NAA), a marker of neuronal integrity, ${ }^{17,18}$ in the dorsolateral prefrontal cortex (DLPFC), anterior cingulate cortex (ACC) and anterior insula in chronic LBP. ${ }^{7-9}$ Further, low NAA in the DLPFC has been correlated with pain severity and associated symptoms, such as anxiety and depression. ${ }^{7,9}$ Another ${ }^{1} \mathrm{H}$-MRS metabolite, myo-inositol (mI), a glial marker, ${ }^{18}$ was also decreased in the ACC and thalamus. ${ }^{10}$ Finally, glutamate, the major brain excitatory neurotransmitter, was also decreased in the ACC. ${ }^{10}$ Taken together, these studies suggested alterations of neuronal-glial interactions in brain regions involved in the affective-motivational aspect of pain. No such studies have been reported that relate to the primary somatosensory cortex (SSC), a major cortical region involved in sensorydiscrimination and nociception integration.

Here, we focus on the SSC, as an extensive reorganization of this area has been reported in chronic LBP. Specifically, an increased activity and an expansion of the back cortical representation into leg and foot representations ${ }^{19}$ were reported in these patients. This finding was also confirmed in people with high catastrophic behavior (illness behavior) and positive Waddell signs following chronic LBP. ${ }^{20}$ Further evidence comes from morphometric studies that showed a decrease in the SSC gray matter volume in chronic LBP. ${ }^{21}$

These SSC functional/morphometric changes are probably accompanied by chemical alterations. Therefore, the goals of the present study were (i) to determine whether ${ }^{1} \mathrm{H}$-MRSvisible metabolites specific to neuronal-glial interactions are altered within the SSC in chronic LBP; and (ii) to investigate whether changes in these metabolites relate to clinical level of pain. Based on the essential role of neuronal-glial interactions in extracellular ionic homeostasis, neurotransmission and ultimately in neuronal function, ${ }^{18}$ we analyzed the following metabolites: NAA; choline (Cho), reflecting cell membrane integrity; mI; and glutamate/glutamine (Glx), reflecting the neuronal-glial neurotransmission system. These measurements were acquired in radiologically normal-appearing grey matter of the SSC.

\section{Methods}

\section{Participants}

Eleven subjects diagnosed with chronic LBP and eleven healthy age-matched subjects participated in the study.
All participants signed informed consent in accordance with the Human Subjects Committee Review Board of the University of Kansas Medical Center. Subjects were included if they (i) had a medical diagnosis of chronic LBP (>3 months), (ii) experienced pain intensity of at least 4 on a verbal numeric pain rating scale (range $0=$ no pain; to $10=$ the worst pain ever experienced), (iii) had no brain abnormalities on T2-weighted MRI, (iv) were aged between 21 and $60 \mathrm{yrs}$, and (v) were able to understand simple instructions in English. Exclusion criteria were: (i) spinal cord compression, tumor, or infection, (ii) psychiatric diseases, (iii) current drug or alcohol abuse, or (iv) other neurological or neuromuscular patologies that might interfere with our data. Five out of eleven patients were on pain therapy. Healthy controls, without neurological, psychiatric or pain disorders, MRI contraindications, and with normal T2-weighted images, were recruited.

\section{Clinical measurements}

Chronic LBP participants completed a physical examination comprised of lumbar range of motion and neurological examination. If the subjects did not present with motor nerve root compression or acute spinal pathology, they proceeded to complete self-reported questionnaires of pain and disability, such as the Short Form McGill Pain Questionnaire (SF-MPQ), Modified Oswestry Disability Scale (MODS), Fear-Avoidance Belief Questionnaire (FABQ), and Back Depression Inventory Scale (BDIS).

SF-MPQ ${ }^{22}$ a reliable and valid scale, ${ }^{23}$ contains 15 pain items, 11 sensory and 4 affective. Each item is rated on an intensity scale with $0=$ none, $1=$ mild, $2=$ moderate, and $3=$ severe. The questionnaire also includes the present pain intensity (PPI) index and a visual analog scale (VAS, $0=$ no pain, $10=$ the worst pain ever experienced) for pain. The MODS, the gold standard test for assessing the disabling effects of lumbar spine pain, consists of 10 sections; each section is scored from $0=$ minimum, $5=$ maximum. Higher scores indicate greater disability. The FABQ has items related to fear about physical (items 2-5) and work (items 6, 7, 9-12, 15) activities and items not in either physical or work scale (items 1, 8, 13, 14, 16); each item is scored from 0 to 6 with a higher number indicating increased fear of activity or work. ${ }^{24}$ The BDIS contains 21 items, each indicating a response from $0=$ does not apply to $3=$ applies very much to me, with the intensity of depression calculated based on the total score. ${ }^{25}$ 


\section{Magnetic resonance imaging (MRI) measurements}

MRI assessments were performed on a 3 Tesla scanner (Siemens Medical Solutions, Erlangen, Germany) and total scan duration was about 30 minutes. A detailed description of these assessments has been reported previously. ${ }^{26}$

In brief, whole-brain 3D T1-weighted MRI was acquired to estimate brain tissue volume in spectroscopic voxels (MPRAGE; TR $=2300 \mathrm{~ms} ; \mathrm{TE}=3 \mathrm{~ms} ; \mathrm{FOV}=240 \mathrm{~mm}$; matrix $=256 \times 256$; resolution $\left.=1 \times 1 \times 1 \mathrm{~mm}^{3}\right)$. An axial proton density/T2-weighted MRI $(\mathrm{TR}=4800 \mathrm{~ms}$; TE1/ TE2 $=18 / 106 \mathrm{~ms} ;$ FOV $=240 \mathrm{~mm}$; matrix size $=256 \times 256$; slice thickness $=5 \mathrm{~mm}$, no gap) was acquired to exclude undiagnosed brain pathology in all participants.

One spectroscopic imaging slab (SIS) was selected in the frontal and parietal lobes sampling both gray matter (GM) and white matter (WM) of our region of interest, the SSC. The SIS was positioned according to the SSC anatomical landmarks ${ }^{27}$ applied on T2-weighted images (Brodmann area 3,1, and 2; or post-central gyrus), and as posteriorly as possible to avoid the poorer magnetic field homogeneity of anterior regions that are not relevant to this study. To minimize the possible lipid artifact, eight outer voxel suppression bands (thickness $=30 \mathrm{~mm}$ ) were prescribed around and above the SIS. SIS was acquired using a pointresolved spectroscopy sequence $(\mathrm{PRESS} ; \mathrm{TE}=30 \mathrm{~ms}$; $\mathrm{TR}=1500 \mathrm{~ms} ;$ matrix size $=16 \times 16 ; \mathrm{FOV}=160 \mathrm{~mm}^{2}$; slice thickness $=15 \mathrm{~mm}$; in-plane resolution $=5 \times 5 \mathrm{~mm}^{2}$; spectral width $=1200 \mathrm{~Hz}$ ). Automated and then manual shimming was performed to achieve full-width at half maximum of $<20 \mathrm{~Hz}$ of the water signal from the entire excitation volume.

Metabolite concentrations were calculated using LCModel $^{28}$ (a linear combination of model spectra using a basis set included in the package), using a radio-frequency coil loading factor. The T1-weighted images were segmented in GM, WM, and cerebrospinal fluid (SPM5, Welcome Department of Cognitive Neurology, London, UK). As previously shown, ${ }^{26}$ by using custom-designed software (Matlab v7.1, 2005), we overlaid the LCModel output and the segmented T1-weighted images on the SIS grid. This graphical interface is used to display spectra, brain fraction from segmentation, and the metabolite concentrations from LCModel in the spectroscopic voxels corresponding to cortical representation of the back in each SSC. Three spectroscopic voxels were selected in each SSC (see Figure 1) and each voxel contained GM $>75 \%$, yielded a signal-to-noise ratio $>10$,

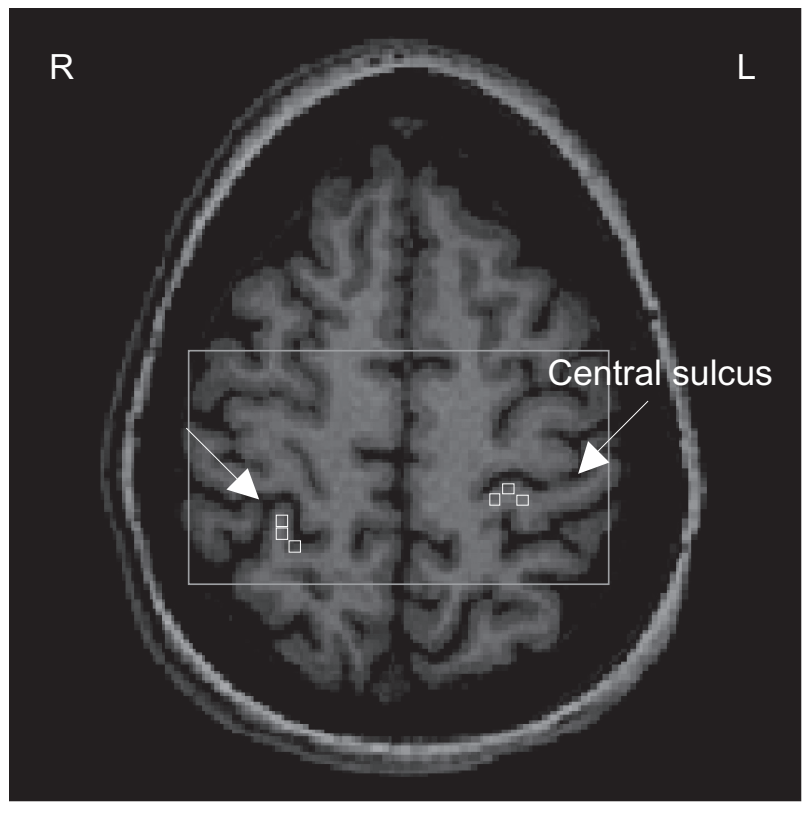

Figure I 'Proton magnetic resonance spectroscopy (H-MRS) analysis: Axial view of the spectroscopic imaging slab (rectangle) superimposed on TI-weighted image. Three spectroscopic voxels (squares) were selected in each somatosensory cortex (SSC). The arrows indicate the central sulcus.

Abbreviations: $R$, right; L, left.

and had Cramer-Rao bounds $<20 \%$ for studied metabolites. Metabolite concentrations were brain tissue-corrected by normalizing the LCModel output as follows: $\mathrm{c}=\mathrm{cLCModel}^{*}[1 /$ $\left.\mathrm{EF}_{\mathrm{BT}}\right]$, where $\mathrm{c}$ is final concentration; $\mathrm{cLCModel}$ is metabolite concentration from LCModel output; and $\mathrm{EF}_{\mathrm{BT}}$ is the estimated brain tissue $(\mathrm{GM}+\mathrm{WM})$ fraction. Finally, the corrected concentrations for all three voxels were averaged to provide a mean concentration (millimoles/kilogram wet weight, $\mathrm{mM}$ ) for NAA, Cho, mI, and Glx in each SSC.

\section{Statistical analysis}

The analysis focused on four metabolites (NAA, Cho, mI, and Glx) in each SSC (left and right) and four clinical measures (pain, SF-MPQ; disability, MODS; fear, FABQ; depression, BDIS). Means and standard deviations were computed for each outcome variable.

The overall differences in metabolite concentrations between groups were analyzed by using independent sample $t$-test with unequal variance (2-tails). Individual metabolite differences between groups were also calculated with independent sample $t$-test ( 2 tails, unequal variance when indicated). Pearson correlation coefficient was used to examine correlations among metabolites within SSC (intraregional) and between left and right SSC (inter-regional). Distribution of original data (correlations strength) for 
goodness of fit was assessed using the K-S test. Using a custom-designed routine (Matlab v7.1, 2005), the computed correlation coefficients were assigned to the following categories $P<0.004$ ( $\mathrm{r}=1-0.77), 0.004<P<0.01$ ( $\mathrm{r}=0.76-0.70), 0.01<P<0.05(\mathrm{r}=0.69-0.61), P>0.05$ $(\mathrm{r}<0.60)$. Similar categories were used for the negative correlations. The respective correlation coefficients were then transformed into gray (for positive) and blue (for negative) values to get a more straightforward visualization of the connectivity between different metabolites (see Figure 2). Pearson correlation coefficient was also used to study the correlations between metabolites and clinical measures. Differences were considered significant at $P<0.05$ (SPSS 18.0, SPSS Inc, Chicago, IL).

\section{Results}

\section{Participants}

LBP and healthy controls did not significantly differ with respect to age (mean $\pm \mathrm{SD}, 33.6 \pm 10.6$ yrs vs $31.4 \pm 13.9$ yrs, $P=0.1$ ). In the LBP group, the mean duration of symptoms was $10.6 \pm 8.3 \mathrm{yrs}$, with an average pain intensity of $5.9 \pm$ 1.4 on VAS. Out of 11 subjects, seven reported radiating leg pain and four reported localized LBP. Five participants were on regular pain therapy (three on opiate analgesics, such as Oxycontin; two on anticonvulsant therapy, such as Neurotin, for pain control). In addition, three subjects took nonsteroidal anti-inflammatory drugs on an as-needed basis (average one medication per week). All but three subjects were carrying fulltime regular work responsibilities. The clinical characteristics of pain, disability, and depression are shown in Table 1. In summary, our subjects suffered long duration of pain symptoms, experienced moderate levels of pain intensity and disability, perceived high levels of fear of movement, and displayed mild depression.

\section{Spectral quality}

${ }^{1} \mathrm{H}$-MRS spectra with good signal-to-noise were obtained consistently from both LBP and controls (left SSC, $11.8 \pm$ 1.6 vs $11.8 \pm 2.0, P=0.9$; right SSC, $12.4 \pm 1.9$ vs $12.3 \pm 2.5$, $P=0.9$ ). Similar percentages of brain tissue within SSC were found between LBP and controls (left, $90.7 \pm 3.9$ vs $87.1 \pm$ $4.8, P=0.1$; right $86.2 \pm 5.4$ vs $82.8 \pm 6.5, P=0.2$ ).

\section{'H-MRS findings}

Normal distribution for individual metabolites was verified with frequency and Q-Q plots, and the assumption of normal distribution was met. Overall metabolite concentrations were lower in both SSCs (left, $-5.0 \%, \mathrm{t}=2.7, P=0.07$; right, $-8.1 \%, \mathrm{t}=4.4, P=0.02)$ in LBP compared to controls. Follow-up analysis showed significantly low NAA in the left SSC and low Cho in the right SSC (see Table 2). $\mathrm{mI}$ and Glx concentrations in either left or right SSC were not statistically different between the groups (Table 2).

The strength of correlations was normally distributed in both groups ( $P=0.02$ for each group). Lower correlations between all metabolites were detected in the right SSC in LBP (mean of correlation coefficients, $0.21 \pm 0.19$ ) than in controls $(0.55 \pm 0.14, \mathrm{t}=2.3, P=0.007$, Figure $2 \mathrm{~A})$. Although the mean correlation coefficient was not altered in the left SSC $(\mathrm{t}=2.2, P=0.1)$, individual correlations between NAA-mI, NAA-Cho and mI-Cho became lower and insignificant in LBP compared to controls $(\mathrm{r}=0.26, P=0.4$ vs $\mathrm{r}=0.83$,

\section{A Intra-regional metabolite correlations}

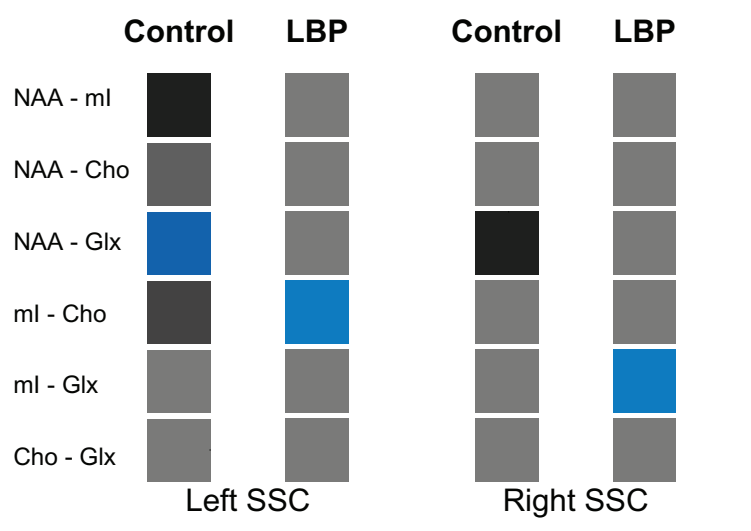

B Inter-regional metabolite correlations

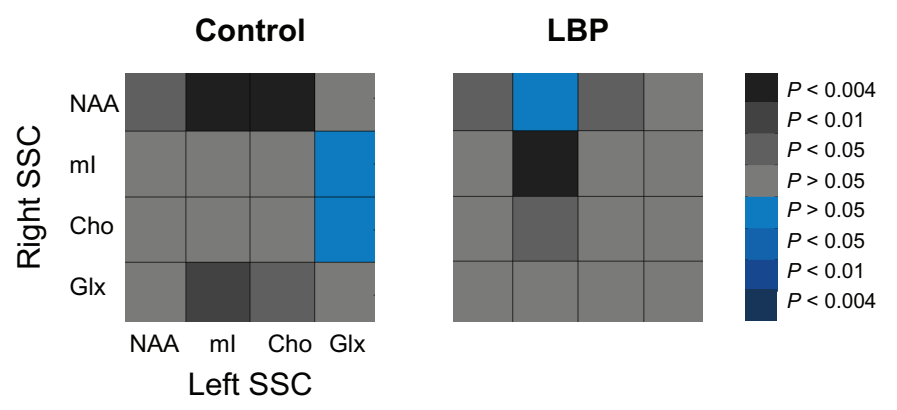

Figure 2 Metabolite connectivity pattern within (A, intra-regional) and between (B, inter-regional) somatosensory cortices (SSCs) in controls (left panels) and chronic low back pain (LBP) patients (right panels). The correlation values (Pearson's correlation coefficients) are presented as color gradients (grey-positive, blue-negative) and the side legend represent the corresponding $P$-values.

Abbreviations: NAA, N-acetylaspartate; ml, myo-inositol; Cho, choline; Glx, glutamate/glutamine. 
Table I Clinical scores (mean \pm SD) in LBP participants

\begin{tabular}{lc}
\hline Clinical scores & \\
\hline SF-MPQ-sensory & $15.1 \pm 6.5$ \\
SF-MPQ-affective & $2.5 \pm 2.2$ \\
SF-MPQ-total & $17.6 \pm 8.0$ \\
SF-PPI & $2.3 \pm 1.0$ \\
MODS-total & $17.3 \pm 12.1$ \\
MODS-\% disability & $34.6 \pm 24.0$ \\
FABQ-physical & $13.5 \pm 5.7$ \\
FABQ-work & $11.6 \pm 11.9$ \\
FABQ-total & $37.5 \pm 28.4$ \\
BDIS & $15.1 \pm 15.6$ \\
\hline
\end{tabular}

Abbreviations: LBP, low back pain; SF-MPQ, Short Form McGill Pain Questionnaire; MODS, Modified Oswestry Disability Scale; FABQ, Fear-Avoidance Belief Questionnaire; BDIS, Back Depression Inventory Scale $(0=$ normal; high score indicates pain, disability, fear of movement or depression).

$P=0.001 ; \mathrm{r}=0.48, P=0.14$ vs $\mathrm{r}=-0.81, P=0.03 ; \mathrm{r}=-0.05$, $P=0.87$ vs $\mathrm{r}=0.73, P=0.01$ respectively; Figure $2 \mathrm{~A}$ ). Further, higher and significant correlations between left $\mathrm{mI}$ and right $\mathrm{Cho}$ and between left and right $\mathrm{mI}$ were detected in LBP compared to controls $(\mathrm{r}=0.61, P=0.05 \mathrm{vs} \mathrm{r}=0.17$, $P=0.6$; and $\mathrm{r}=0.84, P=0.00$ vs $\mathrm{r}=0.50, P=0.1$ respectively; Figure 2B).

Pain duration was negatively correlated with NAA levels in both left $(\mathrm{r}=-0.62, P=0.04)$ and right $(\mathrm{r}=-0.67, P=0.02)$ SSC (Figure 3).

Although Glx concentrations were not significantly changed in LBP compared to controls (see Table 2), the right Glx was positively correlated with pain severity (as measured by SF-MPQ, $\mathrm{r}=0.62, P=0.04)$. No other correlations were found to be significant between SSC metabolites and clinical measures of disability, fear of movement, and depression.

\section{Discussion}

In the present study, two questions were investigated: (i) are cerebral metabolites altered in the SSC in chronic LBP?

Table 2 Metabolite concentrations (mM) in left and right somatosensory cortices (SSCs) in controls and LBP participants

\begin{tabular}{lllll}
\hline & NAA & ml & Cho & Glx \\
\hline Left SSC & & & & \\
Controls & $10.7 \pm 2.0$ & $4.7 \pm 0.7$ & $1.7 \pm 0.2$ & $12.7 \pm 2.7$ \\
LBP & $9.7 \pm 0.5$ & $4.6 \pm 1.0$ & $1.6 \pm 0.6$ & $11.8 \pm 2.4$ \\
$\%$ & -9.0 & -1.5 & -1.8 & -7.5 \\
P-value & 0.001 & 0.84 & 0.88 & 0.42 \\
Right SSC & & & & \\
Controls & $11.1 \pm 1.7$ & $5.2 \pm 0.7$ & $1.8 \pm 0.2$ & $11.9 \pm 3.2$ \\
LBP & $10.4 \pm 1.3$ & $5.0 \pm 1.0$ & $1.6 \pm 0.3$ & $10.8 \pm 2.1$ \\
$\%$ & -6.1 & -4.2 & -12.5 & -9.3 \\
P-value & 0.30 & 0.60 & 0.038 & 0.30 \\
\hline A & & &
\end{tabular}

Abbreviations: LBP, low back pain; NAA, N-acetylasparatate; ml, myo-inositol; Cho, choline; Glx, glutamate/glutamine.

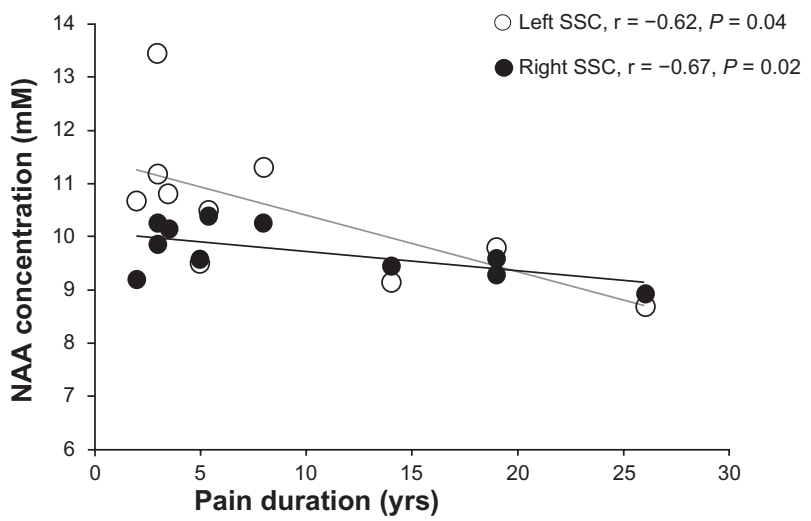

Figure 3 Correlations between $\mathrm{N}$-acetylaspartate (NAA) concentrations (mM) and duration of pain (years) in right (white circles) and left (black circles) somatosensory cortices (SSCs) in participants with low back pain.

(ii) are these alterations related to clinical characteristics of pain? Our results suggest an overall decrease in metabolite levels in the right SSC and to a lesser degree in the left $\mathrm{SSC}$, as well as altered intra- and inter-SSC metabolite correlations in LBP patients compared to controls. In addition, certain SSC metabolites were correlated with pain duration and severity.

Our findings are consistent with previous LBP studies, in which metabolites were decreased overall in cortical regions involved in the affective-motivational aspect of pain. ${ }^{7-9}$ For instance, a decrease of $6.5 \%$ in total metabolite concentrations has been found in the DLPFC. ${ }^{9}$ We also found a similar overall metabolite decrease $(6.5 \%)$ in each SSC in our patients. An anatomical substrate for this overall metabolite change could be the decrease in gray matter volume, as previously reported in these patients. ${ }^{21}$ However, we corrected the metabolite concentrations for brain tissue fraction in each spectroscopic voxel and no significant differences in brain tissue were found between groups (see Results). Therefore, we can conclude that the metabolite alterations described here are not due to decreased grey matter volume in LBP. Finally, our overall SSC metabolite change might underlie the painrelated functional SSC alterations, such as expansion and shift in the representation of low back in the SSC. . $^{1920,29}$ However, no studies have addressed whether the cortical functional reorganization in chronic LBP results from changes in brain chemistry. Such finding will improve our understanding of the neural substrates of chronic pain in humans.

Our finding of low NAA in the left SSC is also in agreement with previous studies. Low NAA have been consistently found in the DLPFC ${ }^{7,9}$ and anterior insula ${ }^{10}$ in 
chronic LBP, as well as in other chronic pain conditions, such as pain following spinal cord injury. ${ }^{30,31}$ Recently, low NAA reported in the hippocampus of individuals who suffered from chronic widespread fibromyalgia pain $^{32,33}$ was attributed to neuronal or axonal metabolic dysfunction. Therefore, the low NAA reported here might indicate altered neuronal mitochondrial metabolism, although other mechanisms such as pain medication or brain atrophy might contribute too. However, we do not attribute our results to medication use since we did not find metabolite changes in both hemispheres. Further, by excluding the five patients taking medications on a regular basis from analysis, in the remaining patients $(n=6)$, NAA was decreased by $11.9 \%$ in the left and $12 \%$ in the right SSC compared to controls. Finally, the presence of the relationships between NAA levels and pain duration (see Figure 3) provide support for NAA as a marker of neuronal mitochondrial depression as a result of long-term nociceptive input to the SSC.

We also report low Cho in the right SSC. Since Cho is thought to be involved in membrane synthesis and degradation, low Cho can be interpreted as an alteration in cell membrane integrity, as indicated by other authors in patients with fibromyalgia pain. ${ }^{32}$ Low Cho might also reflect changes in brain osmolarity. ${ }^{34}$ Since $\mathrm{mI}$, another major brain osmolyte, ${ }^{35}$ was not significantly increased $(P=0.6)$, it is unlikely that low Cho was driven by hyperosmolarity, which would increase mI. Since Cho resonance is composed of choline, phosphocholine and glycerol-phosphocholine, a down-regulation of genes from enzymes responsible for metabolism of free choline, phosphocholine and phosphatidylcholine ${ }^{36}$ could explain the low Cho reported here. However, further studies are needed to determine the exact mechanisms underlying low Cho in chronic pain.

Although no ${ }^{1} \mathrm{H}-\mathrm{MRS}$ measurements have been reported in the $\mathrm{SSC}$, one study reported no metabolite changes in the sensory-motor cortex in chronic LBP. ${ }^{9}$ The use of metabolite ratios to creatine ${ }^{9}$ can partially explain the discrepancy in the individual metabolite findings since creatine is not a reliable denominator. ${ }^{37}$ Indeed, we found significantly low creatine in the left SSC and a trend to low creatine in the right SSC in LBP compared to controls $(6.8 \pm 0.8 \mathrm{mM}$ vs $7.5 \pm 0.6 \mathrm{mM}, P=0.048 ; 10.8 \pm 2.1 \mathrm{mM}$ vs $11.9 \pm 3.2, P=0.3)$. Perhaps a more fundamental reason for the discrepancy is that we studied only the SSC compared to the sensory-motor cortex examined in the previous study. ${ }^{9}$
In contrast to previous studies reporting low ACC Glx in LBP, ${ }^{10}$ we did not find significant Glx changes in the SSC in our LBP patients compared to controls (see Table 2). Although right Glx was not significantly altered in LBP, we found a positive relationship between Glx levels and pain severity. Since glutamate is related to chronic pain sensitization $^{38}$ and it is the major component of Glx, we speculate that Glx might be involved in chronic pain-related SSC changes. However, further studies are required to confirm or reject this finding.

Since the SSC is involved in the sensory discrimination process of pain rather than the affective-motivational pain aspect, as expected, we did not find significant correlations between metabolite concentrations and the clinical measures of fear avoidance and depression.

The neuronal network exists through a close relationship among various metabolites at physiological levels; a relationship that can be disturbed in pathologies. For example, the metabolite correlations within the DLPFC, ACC and thalamus were altered in chronic LBP. ${ }^{9}$ In agreement with these findings, we report weaker (or depression in) correlations between all metabolites in both SSC, predominantly in the right (see Figure 2A), in our patients than in the controls. Indeed, right SSC shows important changes of Cho, $\mathrm{mI}$, and Glx concentrations (relative to controls, see Table 2), which might affect the correlation strengths between metabolites in this region. In contrast, stronger and significant correlations were found across hemispheres, specifically between left $\mathrm{mI}$ and right $\mathrm{mI}$ and between left $\mathrm{mI}$ and right Cho (see Figure 2B). This inter-hemispheric increased metabolite connectivity may be considered as an adaptive process, which might be necessary to preserve optimal SSC metabolism when metabolite concentrations are low. These findings highlight the dynamic nature of changes not only in metabolite concentrations but also in their "communication" in chronic pain.

A limitation of our study is that we studied a small sample size $(n=11)$, and therefore we did not control for multiple comparisons, as in any pilot study. Despite this limitation, we observed changes in several brain metabolites as well as alterations in metabolite correlations that may potentially explain pain persistence.

\section{Conclusion}

In summary, we report the presence of SSC metabolite alterations, reflective of altered neuronal-glial interactions that correlate with clinical characteristics of pain. These changes may contribute to the persistent nature of pain 
without obvious pathology. Our results add to the current understanding of the neural substrate of cortical sensitization in chronic pain.

\section{Acknowledgments}

This work was supported by the following funding sources: American Heart Association (0860041Z to CMC), KUMC Clinical Pilot and Lied Endowed Basic Science Program (to NKS), and 5 T32 HD57850 (to AL). The Hoglund Brain Imaging Center is supported by a generous gift from Forrest and Sally Hoglund. The authors like to thank Ali Bani-Ahmed and Anda Popescu for their assistance in data acquisition and analysis.

\section{Disclosure}

The authors report no conflicts of interest in this work.

\section{References}

1. Andersson GB. Epidemiological features of chronic low-back pain. Lancet. 1999;354(9178):581-585.

2. Ritzwoller DP, Crounse L, Shetterly S, Rublee D. The association of comorbidities, utilization and costs for patients identified with low back pain. BMC Musculoskelet Disord. 2006;7:72.

3. Wasiak R, Kim J, Pransky G. Work disability and costs caused by recurrence of low back pain: longer and more costly than in first episodes. Spine (Phila Pa 1976). 2006;31(2):219-225.

4. Martin BI, Deyo RA, Mirza SK, et al. Expenditures and health status among adults with back and neck problems. JAMA. 2008;299(6): 656-664.

5. Jensen MC, Brant-Zawadzki MN, Obuchowski N, Modic MT, Malkasian D, Ross JS. Magnetic resonance imaging of the lumbar spine in people without back pain. $N$ Engl J Med. 1994;331(2):69-73.

6. Giesecke T, Gracely RH, Grant MA, et al. Evidence of augmented central pain processing in idiopathic chronic low back pain. Arthritis Rheum. 2004;50(2):613-623.

7. Grachev ID, Ramachandran TS, Thomas PS, Szeverenyi NM, Fredrickson BE. Association between dorsolateral prefrontal n-acetyl aspartate and depression in chronic back pain: an in vivo proton magnetic resonance spectroscopy study. J Neural Transm. 2003;110(3): 287-312.

8. Grachev ID, Fredrickson BE, Apkarian AV. Brain chemistry reflects dual states of pain and anxiety in chronic low back pain. $J$ Neural Transm. 2002;109(10):1309-1334.

9. Grachev ID, Fredrickson BE, Apkarian AV. Abnormal brain chemistry in chronic back pain: an in vivo proton magnetic resonance spectroscopy study. Pain. 2000;89(1):7-18.

10. Gussew A, Rzanny R, Gullmar D, Scholle HC, Reichenbach JR. 1h-mr spectroscopic detection of metabolic changes in pain processing brain regions in the presence of non-specific chronic low back pain. Neuroimage. 2010;54(2):1315-1323.

11. Wrigley PJ, Press SR, Gustin SM, et al. Neuropathic pain and primary somatosensory cortex reorganization following spinal cord injury. Pain 2009;141(1-2):52-59.

12. Henderson LA, Gustin SM, Macey PM, Wrigley PJ, Siddall PJ. Functional reorganization of the brain in humans following spinal cord injury: evidence for underlying changes in cortical anatomy. J Neurosci. 2011;31(7):2630-2637.

13. Sharma NK, Ryals JM, Liu H, Liu W, Wright DE. Acidic saline-induced primary and secondary mechanical hyperalgesia in mice. $J$ Pain 2009;10(12):1231-1241.
14. Cui JG, O'Connor WT, Ungerstedt U, Linderoth B, Meyerson BA. Spinal cord stimulation attenuates augmented dorsal horn release of excitatory amino acids in mononeuropathy via a gabaergic mechanism. Pain. 1997;73(1):87-95.

15. Bement MK, Sluka KA. Co-localization of p-creb and p-nr1 in spinothalamic neurons in a chronic muscle pain model. Neurosci Lett. 2007;418(1):22-27.

16. Tillu DV, Gebhart GF, Sluka KA. Descending facilitatory pathways from the rvm initiate and maintain bilateral hyperalgesia after muscle insult. Pain. 2008;136(3):331-339.

17. Govindaraju V, Young K, Maudsley AA. Proton NMR chemical shifts and coupling constants for brain metabolites. NMR Biomed. 2000;13(3):129-153.

18. Siegel GJB, Agranoff BW, Albers GW, Fisher SK, Uhler MD. Basic Neurochemistry: Molecular, Cellular and Medical Aspects. Oxford, UK: Academic Press; 2005.

19. Flor H, Braun C, Elbert T, Birbaumer N. Extensive reorganization of primary somatosensory cortex in chronic back pain patients. Neurosci Lett. 1997;224(1):5-8.

20. Lloyd D, Findlay G, Roberts N, Nurmikko T. Differences in low back pain behavior are reflected in the cerebral response to tactile stimulation of the lower back. Spine (Phila Pa 1976). 2008;33(12): 1372-1377.

21. Schmidt-Wilcke T, Leinisch E, Ganssbauer S, et al. Affective components and intensity of pain correlate with structural differences in gray matter in chronic back pain patients. Pain. 2006;125(1-2):89-97.

22. Melzack R. The short-form McGill pain questionnaire. Pain. 1987;30(2): 191-197.

23. Zinke JL, Lam CS, Harden RN, Fogg L. Examining the cross-cultural validity of the English short-form McGill pain questionnaire using the matched moderated regression methodology. Clin J Pain. 2010; 26(2):153-162.

24. Waddell G, Newton M, Henderson I, Somerville D, Main CJ. A fearavoidance beliefs questionnaire (FABQ) and the role of fear-avoidance beliefs in chronic low back pain and disability. Pain. 1993;52(2): 157-168.

25. Beck AT, Steer RA. Internal consistencies of the original and revised Beck depression inventory. J Clin Psychol. 1984;40(6):1365-1367.

26. Cirstea MC, Brooks WM, Craciunas SC, et al. Primary motor cortex in stroke - a functional MRI-guided proton MR spectroscopic study. Stroke. 2011;42(4):1004-1009.

27. Constable RT, Skudlarski P, Mencl E, et al. Quantifying and comparing region-of-interest activation patterns in functional brain MR imaging: methodology considerations. Magn Reson Imaging. 1998;16(3): 289-300.

28. Provencher SW. Estimation of metabolite concentrations from localized in vivo proton NMR spectra. Magn Reson Med. 1993;30(6): 672-679.

29. Moseley GL. Widespread brain activity during an abdominal task markedly reduced after pain physiology education: FMRI evaluation of a single patient with chronic low back pain. Aust J Physiother. 2005; 51(1):49-52.

30. Fukui $\mathrm{S}$, Matsuno $\mathrm{M}$, Inubushi $\mathrm{T}$, Nosaka S. N-acetylaspartate concentrations in the thalami of neuropathic pain patients and healthy comparison subjects measured with (1)H-MRS. Magn Reson Imaging. 2006;24(1):75-79.

31. Pattany PM, Yezierski RP, Widerstrom-Noga EG, et al. Proton magnetic resonance spectroscopy of the thalamus in patients with chronic neuropathic pain after spinal cord injury. AJNR Am J Neuroradiol. 2002;23(6):901-905.

32. Fayed N, Garcia-Campayo J, Magallon R, et al. Localized 1H-NMR spectroscopy in patients with fibromyalgia: a controlled study of changes in cerebral glutamate/glutamine, inositol, choline, and n-acetylaspartate. Arthritis Res Ther. 2010;12(4):R134.

33. Wood PB, Ledbetter CR, Glabus MF, Broadwell LK, Patterson JC 2nd. Hippocampal metabolite abnormalities in fibromyalgia: correlation with clinical features. J Pain. 2009;10(1):47-52. 
34. Bosman DK, Deutz NE, De Graaf AA, et al. Changes in brain metabolism during hyperammonemia and acute liver failure: results of a comparative 1H-NMR spectroscopy and biochemical investigation. Hepatology. 1990;12(2):281-290.

35. Heilig CW, Stromski ME, Blumenfeld JD, Lee JP, Gullans SR. Characterization of the major brain osmolytes that accumulate in saltloaded rats. Am J Physiol. 1989;257(6 Pt 2):F1108-F1116.

36. Li Z, Vance DE. Phosphatidylcholine and choline homeostasis. J Lipid Res. 2008;49(6):1187-1194.
37. Laakso MP, Hiltunen Y, Kononen M, et al. Decreased brain creatine levels in elderly apolipoprotein e epsilon 4 carriers. J Neural Transm. 2003;110(3):267-275.

38. Harris RE, Sundgren PC, Craig AD, et al. Elevated insular glutamate in fibromyalgia is associated with experimental pain. Arthritis Rheum. 2009;60(10):3146-3152.

\section{Publish your work in this journal}

The Journal of Pain Research is an international, peer-reviewed, open access, online journal that welcomes laboratory and clinical findings in the fields of pain research and the prevention and management of pain. Original research, reviews, symposium reports, hypothesis formation and commentaries are all considered for publication.

\section{Dovepress}

The manuscript management system is completely online and includes a very quick and fair peer-review system, which is all easy to use. Visit http://www.dovepress.com/testimonials.php to read real quotes from published authors. 